\title{
Lax Pair, Hirota Bilinear Form and Soliton Solutions of the Seventh-Order Kaup-Kupershmidt Equation in Plasma Physics
}

\author{
Yuping Zhang a , Wenguang Zhang ${ }^{b}$ \\ School of Computer Science and Engineering, Beihang University, Beijing 100191, China \\ azhyuping@buaa.edu.cn, bzhangwg1990@126.com
}

Keywords: Seventh-order KK equation, Lax pair, Hirota bilinear form, Soliton solution.

\begin{abstract}
In this paper, the seventh-order Kaup-Kupershmidt (KK) equation arising in fluids and plasmas is considered. The pseudopotentials of the equation are derived, based on the pseudopotentials, the Lax pair of the equation is get, which shows that the equation has the Lax integrability. It is a very important property in researching nonlinear evolution equations (NLEEs). By virtue of the symbolic computation, the equation is transformed into its bilinear form and soliton solutions of the equation are obtained.
\end{abstract}

\section{Introduction}

Integrability plays an important role in studying of NLEEs [1]. Lax pair, which is a kind of integrability of NLEEs, can be regarded as a predictor of the equation's complete integrability.

In nonlinear theory, the Korteweg-de Vries (KdV) type equations are very important since they can model many physical phenomena such as wave propagation in the ocean, stratified internal waves, ion-acoustic waves and so on [2]. The KK equations, as a kind of KdV type equations, are of great value to research. In this paper, we will investigate the Seven-order KK equation,

$$
u_{t}+2016 u^{3} u_{x}+630 u_{x}^{3}+2268 u u_{x} u_{2 x}+504 u^{2} u_{3 x}+252 u_{2 x} u_{3 x}+147 u_{x} u_{4 x}+42 u u_{5 x}+u_{7 x}=0 \text {, }
$$

Where $u$ is a real function of $x$ and $t$. With another group of parameters, there is the Sawada-Kotera-Ito equation,

$$
u_{t}+252 u^{3} u_{x}+63 u_{x}^{3}+378 u u_{x} u_{2 x}+126 u^{2} u_{3 x}+63 u_{2 x} u_{3 x}+42 u_{x} u_{4 x}+21 u u_{5 x}+u_{7 x}=0 .
$$

$\mathrm{N}$-soliton solutions, bilinear form and Lax pair for equation (2) have been given [3], and the Bell-polynomial approach is extended to the equation.

Different from the research method to equation (2), the poseudopotential procedure will be, hereby introduced to deal with equation (1). In section 2, the Lax pair of equation (1) will be constructed from poseudopotentials. Then in section 3 base on the Lax pair, the bilinear form of equation (1) will be derived and the soliton solutions will be given.

\section{Poseudopotential and Lax pair}

If we can find the Lax pair of an NLEE, then we can say that the equation is complete integrable. It is very useful for further research of the equation.

In this section, we will construct the Lax pair of equation (1) by means of the method developed by Nucci [4]. Nucci considered the Riccati-type pseudopotentials of NLEEs, and extended them to derive Lax pairs, auto-Bäcklund transformations, and singularity manifold equations. Afterwards this method was extended to investigate many NLEEs.

For equation (1), we assume there exists a pseudopotential with the form,

$$
\begin{aligned}
& q_{x}=k q^{2}+F_{1}(u) q+F_{0}(u), \\
& q_{t}=G\left(q, u, u_{x}, u_{2 x}, u_{3 x}, u_{4 x}, u_{5 x}, u_{6 x}, u_{7 x}, u_{8 x}\right),
\end{aligned}
$$

Where $\mathrm{G}$ is a second-order function in $q$, and $k$ is a constant. If we can get the pseudopotential of equation (1), we can construct the Lax pairs and the auto-Bäcklund transformation.

Based on the compatible condition $q_{x t}=q_{t x}$, we obtain the poseudopotential of equation (1) 


$$
\begin{aligned}
& q_{x}=\frac{\gamma^{2}}{4 k}+\gamma q+k q^{2}+\frac{3}{2} u, \\
& q_{t}=-\frac{3}{2 k}\left[\left(96 u^{3}+18 u_{x}^{2}+36 u u_{2 x}+u_{4 x}\right)(\gamma+2 k q)+288 u^{2} u_{x}+72 u_{x} u_{2 x}+36 u u_{3 x}+u_{5 x}\right]_{x},
\end{aligned}
$$

Where $\gamma$ is a spectral parameter. Then applying the transformation $q=-(\ln \psi)_{x}$, the pseudopotential system (5) and (6) can be linearized to the following system,

$$
\begin{aligned}
\psi_{x x}= & -\frac{1}{4}\left(\gamma^{2}+6 u\right) \psi+\gamma \psi_{x}, \\
\psi_{t}= & \frac{3 \psi}{2 k}\left[96 \gamma u^{3}+288 u^{2} u_{x}+18 \gamma u_{x}^{2}+72 u_{x} u_{2 x}+36 u\left(\gamma u_{2 x}+u_{3 x}\right)+\gamma u_{4 x}+u_{5 x}\right] \\
& -\frac{3 \psi_{x}}{k}\left(96 u^{3}+18 u_{x}^{2}+36 u u_{2 x}+u_{4 x}\right) .
\end{aligned}
$$

With the transformation $q=\frac{\phi_{1}}{\phi_{2}}$ and $\gamma=2 \lambda$, we can get the Lax pair of equation (1) in the Ablowitz-Kaup-Newell-Segur (AKNS) form,

$$
\begin{aligned}
& \Phi_{x}=U \Phi=\left(\begin{array}{cc}
\lambda & \lambda^{2}+\frac{3 u}{2 k} \\
-k & -\lambda
\end{array}\right) \Phi, \\
& \Phi_{t}=V \Phi=\left(\begin{array}{cc}
A(x, t, \lambda) & B(x, t, \lambda) \\
C(x, t, \lambda) & -A(x, t, \lambda)
\end{array}\right) \Phi,
\end{aligned}
$$

where

$$
\begin{aligned}
A(x, t, \lambda)= & -\left[\left(576 u^{3}+108 u_{x}^{2}-216 u u_{2 x}-6 u_{4 x}\right) \lambda+864 u^{2} u_{x}+216 u_{x} u_{2 x}+108 u u_{3 x}-3 u_{5 x}\right] / 2, \\
B(x, t, \lambda)=- & {\left[\left(288 u^{3}+54 u_{x}^{2}+108 u u_{2 x}-3 u_{4 x}\right) \lambda^{2}+\left(864 u^{2} u_{x}+216 u_{x} u_{2 x}+108 u u_{3 x}+3 u_{5 x}\right) \lambda\right.} \\
& \left.+432 u^{4}+945 u u_{x}^{2}+594 u^{2} u_{2 x}+108 u_{2 x}^{2}+162 u_{x} u_{3 x}+117 u u_{4 x} / 2+3 u_{6 x}\right] / k, \\
C(x, t, \lambda)= & 288 k u^{3}+54 k u_{x}^{2}+108 k u u_{2 x}+3 k u_{4 x} .
\end{aligned}
$$

It can be verified that the compatibility condition $q_{x t}=q_{t x}$ with equation (11) to equation (13) leads to equation (1), which ensured the correction of the Lax pair we obtained.

\section{Bilinear form and soliton solutions}

In this section, we first derive the bilinear representation of equation (1), and then construct it's multi-soliton solutions using Hirota's bilinear method [5].

Integrate equation (1) with respect to $x$, we have

$$
\int u_{t} \mathrm{~d} x+504 u^{4}+630 u u_{x}^{2}+504 u^{2} u_{2 x}+\frac{147}{2} u_{2 x}^{2}+105 u_{x} u_{3 x}+42 u u_{4 x}+u_{6 x}=0 \text {. }
$$

Based on Painlevé expansion, the transformation can be written as

$$
u=\frac{1}{2}(\ln f)_{2 x}+u_{0} \text {, }
$$

Where $u_{0}$ is a solution of equation (1) and $f$ is a function of $x$ and $t$. Here we take the trivial solution $u_{0}=0$. Substituting transformation (15) into equation (1), we have 


$$
\begin{aligned}
\frac{f_{x t} f-f_{x} f_{t}}{2 f^{2}} & +\frac{1}{8 f^{2}}\left(4 f_{8 x} f-32 f_{x} f_{7 x}-28 f_{2 x} f_{5 x}-14 f_{3 x} f_{5 x}+7 f_{4 x}^{2}\right) \\
& +\frac{7}{4 f^{3}}\left(3 f_{2 x}^{2} f_{4 x}-5 f_{2 c} f_{3 x}^{2}+15 f_{x} f_{2 x} f_{5 x}+f_{x} f_{3 x} f_{4 x}+10 f_{x}^{2} f_{6 x}\right) \\
& +\frac{105}{4 f^{6}}\left(3 f_{x}^{4} f_{2 x}^{2}-4 f_{x}^{5} f_{3 x}\right)++\frac{105}{4 f^{5}}\left(4 f_{x}^{4} f_{4 x}-3 f_{x}^{2} f_{2 x}^{3}+2 f_{x}^{3} f_{2 x} f_{3 x}\right) \\
& +\frac{21}{8 f^{4}}\left(3 f_{2 x}^{4}+12 f_{x} f_{2 x}^{3} f_{3 x}+2 f_{x}^{2} f_{3 x}^{2}-30 f_{x}^{2} f_{2 x} f_{4 x}-20 f_{x}^{3} f_{5 x}\right)=0 .
\end{aligned}
$$

Introducing an auxiliary function $g$ defined as

$$
\frac{g}{f}=\frac{D_{x}^{4} f \cdot f}{64 f^{2}}
$$

We can obtain the bilinear form of equation (1) as bellow

$$
\begin{aligned}
& {\left[D_{x} D_{t}-\frac{1}{64} D_{x}^{8}\right] f \cdot f-65 D_{x}^{4} f \cdot g=0,} \\
& D_{x}^{4} f \cdot f+64 f \cdot g=0,
\end{aligned}
$$

Where the bilinear operator $\mathrm{D}$ is defined by

$$
D_{x}^{m} D_{t}^{n} f(x, t) \cdot g(x, t)=\frac{\partial^{m}}{\partial r^{m}} \frac{\partial^{n}}{\partial s^{n}} \times\left. f(x+r, t+s) g(x-r, t-s)\right|_{r=0, s=0}, \quad m, n=1,2, \cdots
$$

To find the one-soliton solution, we make the ansatz

$$
\begin{aligned}
& f=1+\epsilon e^{\eta_{1}}+\epsilon^{2} f_{2}, \\
& \eta_{1}=k_{1} x+\omega_{1} t+\xi_{1}^{0}, \\
& g=\epsilon g_{1} .
\end{aligned}
$$

Substituting (21) into (18) and (19), we have

$$
\begin{aligned}
& \omega_{1}=-k_{1}^{7}, \\
& g_{1}=-\frac{k_{1}^{4} e^{\eta_{1}}}{32}, \\
& f_{2}=\frac{e^{2 \eta_{1}}}{16},
\end{aligned}
$$

And with some symbolic computation, we get the one-soliton solution

$u=\frac{8 k_{1}^{2} e^{\eta_{1}}\left(16+4 e^{\eta_{1}}+e^{2 \eta_{1}}\right)}{\left(16+16 e^{\eta_{1}}+e^{2 \eta_{1}}\right)^{2}}$

Where $\eta_{1}=k_{1} x-k_{1}^{7} t+\xi_{1}^{0}, \quad k_{1}$ and $\xi_{1}^{0}$ are arbitrary real constants.

To get the two-soliton solutions, we assume

$$
\begin{aligned}
& f=1+\epsilon e^{\eta_{1}+\eta_{2}}+\epsilon^{2} f_{2}+\epsilon^{3} f_{3}+\epsilon^{4} f_{4}, \\
& g=\epsilon g_{1}+\epsilon^{2} g_{2}+\epsilon^{3} g_{3},
\end{aligned}
$$

Where $\eta_{i}=k_{i} x+\omega_{i} t+\xi_{i}^{0}(i=1,2)$. Substituting equation (24) into the bilinear form equation (18) and equation (19), we obtain 


$$
\begin{aligned}
& \omega_{1}=-k_{1}^{7}, \\
& \omega_{2}=-k_{2}^{7}, \\
& f_{2}=\frac{1}{16} e^{2 \eta_{1}}+\frac{1}{16} e^{2 \eta_{2}}+\mu_{12} e^{\eta_{1}+\eta_{2}}, \\
& f_{3}=\sigma_{12}\left(e^{2 \eta_{1}+\eta_{2}}+e^{\eta_{1}+2 \eta_{2}}\right), \\
& f_{4}=\sigma_{12} e^{2 \eta_{1}+2 \eta_{2}},
\end{aligned}
$$

where

$$
\begin{aligned}
& \mu_{12}=\frac{2 k_{1}^{4}+2 k_{2}^{4}-k_{1}^{2} k_{2}^{2}}{2\left(k_{1}+k_{2}\right)^{2}\left(k_{1}^{2}+k_{1} k_{2}+k_{2}^{2}\right)}, \\
& \sigma_{12}=\frac{\left(k_{1}-k_{2}\right)^{2}\left(k_{1}^{2}+k_{2}^{2}-k_{1} k_{2}\right)}{16\left(k_{1}+k_{2}\right)^{2}\left(k_{1}^{2}+k_{2}^{2}+k_{1} k_{2}\right)} .
\end{aligned}
$$

In the same way, we get the two-soliton solution as

$u=\frac{1}{2}(\ln f)_{2 x}$,

where $f$ and $\eta_{j}$ is given by

$$
\begin{aligned}
f= & 1+e^{\eta_{1}}+e^{\eta_{2}}+\frac{e^{2 \eta_{1}}}{16} \\
& +\frac{e^{2 \eta_{2}}}{16}+\mu_{12} e^{\eta_{1}+\eta_{2}} \\
& +\sigma_{12}\left(e^{2 \eta_{1}+\eta_{2}}+e^{\eta_{1}+2 \eta_{2}}\right)+\sigma_{12}^{2} e^{2 \eta_{1}+2 \eta_{2}}, \\
\eta_{j} & =k_{j} x-k_{j}^{7} t+\xi_{j}^{0}, j=1,2 .
\end{aligned}
$$

\section{Conclusion}

Seventh-order Kaup-Kupershmidt (KK) equation arising in fluids and plasmas was considered in this paper. With symbol computation, Lax pair in AKNS form are given by poseudopotential. The bilinear form of the equation is found by Lax pair. Then based on the bilinear, the soliton solutions are obtained.

\section{References}

[1]. Y. P. Zhang, J. Y. Wang. The Painlevé property, Bäcklund transformation, Lax pair and new analytic solutions of a generalized variable-coefficient $\mathrm{KdV}$ equation from fluids and plasmas. Physica Scripta. Vol. 90 (2015) No. 6, p. 065203.

[2]. Y. P. Zhang, J. Liu, G. M. Wei. Lax pair, auto-Bäcklund transformation and conservation law for a generalized variable-coefficient $\mathrm{KdV}$ equation with external-force term. Applied Mathematics Letters. Vol. 45 (2015), p. 58-63.

[3]. Ito M. An extension of nonlinear evolution equations of the K-dV (mK-dV) type to higher orders. Journal of the Physical Society of Japan, Vol. 49(1980) No. 2, p. 771-778.

[4]. M.C. Nucci. Poseudopotentials, Lax equations and Bäcklund transformations for non-linear evolution equations, Journal of Physics A: Mathematical and General. Vol. 21(1988), p. 73-79.

[5]. W. X. Ma, Y. C. You. Solving the Korteweg-de Vries equation by its bilinear form: Wronskian solutions. Transactions of the American mathematical society, Vol. 357(2005) No. 5, p. 1753-1778. 